\title{
SADDLE POINT CRITERIA AND DUALITY IN MULTIOBJECTIVE PROGRAMMING VIA AN $\eta$-APPROXIMATION METHOD
}

\author{
TADEUSZ ANTCZAK ${ }^{1}$
}

(Received 12 July, 2004; revised 5 January, 2005)

\begin{abstract}
In this paper, Antczak's $\eta$-approximation approach is used to prove the equivalence between optima of multiobjective programming problems and the $\eta$-saddle points of the associated $\eta$-approximated vector optimisation problems. We introduce an $\eta$-Lagrange function for a constructed $\eta$-approximated vector optimisation problem and present some modified $\eta$-saddle point results. Furthermore, we construct an $\eta$-approximated Mond-Weir dual problem associated with the original dual problem of the considered multiobjective programming problem. Using duality theorems between $\eta$-approximation vector optimisation problems and their duals (that is, an $\eta$-approximated dual problem), various duality theorems are established for the original multiobjective programming problem and its original Mond-Weir dual problem.
\end{abstract}

\section{Introduction}

Saddle point criteria and duality results in multiobjective programming problems have attracted much interest in recent years. The concept of a (weakly) efficient point (a (weak) Pareto optimal point) [15] has played useful roles in the analysis and solutions of this type of optimisation problem.

Weir [18] proved weak and strong duality theorems for a Mond-Weir [14] dual of a primal multiobjective programming problem. In his strong duality theorem he obtained an efficient solution of the dual from a properly efficient solution of the primal multiobjective programming problem. Bector et al. [5] also discussed a similar result under stronger convexity assumptions. The duality results in Singh [16] and Weir and Mond [20] are for efficient and weak efficient solutions, respectively. The converse duality theorems in [5] and [16] are proved using the Kuhn-Tuckertype necessary conditions of Singh [17] and therefore need a constraint qualification.

\footnotetext{
'Faculty of Mathematics, University of Łódź, Banacha 22, 90-238 Łódź, Poland; e-mail: antczak@math.uni.lodz.pl.

(C) Australian Mathematical Society 2005, Serial-fee code 1446-1811/05
} 
The strong duality theorem of Gulati and Talaat [10] provides a properly efficient solution of the dual while in their converse duality theorem a weak efficient solution of the dual gives a properly efficient solution of the primal multiobjective programming problem. Moreover, their converse duality theorem is proved using F. John's necessary conditions which do not need a constraint qualification.

The convex problem has been extensively studied in recent years, and various generalisations have been successfully applied to efficiency and weak efficiency necessary conditions, alternative theorems, multiplier rules, duality results, and so on.

In [11], Hanson extended the optimality conditions and duality results of mathematical programming to a class of functions which were subsequently called invex by Craven [6].

Several authors have studied the theory of multiobjective saddle point criteria and duality results for invex functions. For example, Egudo and Hanson [8] have studied a multiobjective programming problem with Mond-Weir-type and Wolfe-type duals for invex objective and quasi-invex constraint functions. Weir [18] considered a multiobjective programming problem which involved invex functions and obtained Karush-Kuhn-Tucker-type necessary and sufficient conditions for a feasible point to be properly efficient. Kim [12] proved optimality conditions and duality results for differentiable multiobjective programming problems under invexity assumptions.

Considerable attention has been given recently to devising new methods which solve the original multiobjective mathematical programming problem and its duals with the help of some associated vector optimisation problems.

One such method is that proposed by Antczak [1]. He introduced a new approach with a modified objective function for solving differentiable multiobjective optimisation problems involving invex functions. He obtained optimality conditions for Pareto optimality by constructing an equivalent vector minimisation problem with a modified objective function for a considered multiobjective programming problem and then using an invexity concept in multiobjective programming.

Later, Antczak [3] introduced an $\eta$-approximation method for solving a differentiable multiobjective programming problem. This method is an extension of an approach with a modified objective function.

The purpose of this paper is to apply the $\eta$-approximation method to develop saddle point criteria and duality theory for differentiable multiobjective programming problems involving invex functions with respect to the function $\eta$. An equivalent associated $\eta$-approximated vector optimisation problem is obtained in this method by a modification of both the objective and constraint functions in the given multiobjective programming problem at an arbitrary but fixed point $\bar{x}$. In this paper, a definition of the $\eta$-Lagrange function in such vector optimisation problems is given, for which modified vector-valued saddle point results are presented. The equivalence between optima of the original multiobjective programming problem and $\eta$-saddle points of its 
associated $\eta$-approximated vector optimisation problem is proved under an invexity assumption.

Furthermore, we show how to obtain duality results for a differentiable multiobjective programming problem by using the $\eta$-approximation method proposed by Antczak [3]. Based on this approach, we construct the $\eta$-approximated Mond-Weir vector dual problem for an original Mond-Weir dual problem of the considered multiobjective programming problem. It turns out that the constructed $\eta$-approximated vector for the Mond-Weir dual problem is the dual for the $\eta$-approximated vector optimisation problem associated with the original multiobjective programming problem. Using proved duality results between the $\eta$-approximated vector optimisation problem and its Mond-Weir dual problems (that is, its $\eta$-approximated Mond-Weir duals), we establish various duality results between the original multiobjective programming problem and its original Mond-Weir duals.

\section{Preliminaries}

The following convention for equalities and inequalities will be used throughout the paper.

For any $x=\left(x_{1}, x_{2}, \ldots, x_{n}\right)^{T}, y=\left(y_{1}, y_{2}, \ldots, y_{n}\right)^{T}$, we define:

(i) $x=y$ if and only if $x_{i}=y_{i}$ for all $i=1,2, \ldots, n$;

(ii) $x<y$ if and only if $x_{i}<y_{i}$ for all $i=1,2, \ldots, n$;

(iii) $x \leqq y$ if and only if $x_{i} \leqq y_{i}$ for all $i=1,2, \ldots, n$;

(iv) $x \leq y$ if and only if $x \leqq y$ and $x \neq y$;

(v) $x \nless y$ is the negation of $x<y$.

To make things easier, we consider the invexity definitions for vectorial functions, which coincide with those given in the scalar case (see [11]).

DEFINITION 2.1. Let $f: X \rightarrow R^{k}$ be a differentiable function on a nonempty set $X \subset R^{n}$. Then $f$ is invex with respect to $\eta$ at $u \in X$ on $X$ if, for all $x \in X$, there exists an $\eta: X \times X \rightarrow R^{n}$ such that

$$
f(x)-f(u) \geqq \nabla f(u) \eta(x, u) .
$$

If inequality (2.1) holds for any $u \in X$ then $f$ is invex with respect to $\eta$ on $X$.

DEFINITION 2.2. Let $f: X \rightarrow R^{k}$ be a differentiable function on a nonempty set $X \subset R^{n}$. Then $f$ is strictly invex with respect to $\eta$ at $u \in X$ on $X$ if, for all $x \in X$ with $x \neq u$, there exists an $\eta: X \times X \rightarrow R^{n}$ such that

$$
f(x)-f(u)>\nabla f(u) \eta(x, u) .
$$

If inequality (2.2) holds for any $u \in X$ then $f$ is strictly invex with respect to $\eta$ on $X$. 
We consider the following multiobjective programming problem:

$$
\begin{aligned}
V \text {-Minimise } & f(x):=\left(f_{1}(x), \ldots, f_{k}(x)\right) \\
\text { subject to } & g(x):=\left(g_{1}(x), \ldots, g_{m}(x)\right) \leqq 0,
\end{aligned}
$$

where $f: X \rightarrow R^{k}$ and $g: X \rightarrow R^{m}$ are differentiable functions on a nonempty open set $X \subset R^{n}$. Note here that the symbol " $V$-Minimise" stands for vector minimisation. This is the problem of finding the set of weak minima (see Definition 2.4) for the considered vector optimisation problem (VP). We will define (VP) as the original multiobjective optimisation problem.

Let $D:=\left\{x \in X: g_{j}(x) \leqq 0, j=1, \ldots, m\right\}$ denote the set of all feasible solutions of (VP).

We define a Lagrange function for the original multiobjective programming problem (VP) to be

$$
L(x, \lambda, \xi):=\lambda f(x)+\xi g(x) e=\left(\lambda_{1} f_{1}(x)+\xi g(x), \ldots, \lambda_{k} f_{k}(x)+\xi g(x)\right),
$$

where $\lambda \in R_{+}^{k}, \xi \in R_{+}^{m}$ and $e=(1, \ldots, 1) \in R^{k}$.

For such multicriterion optimisation problems, the solution is defined in terms of a (weak) Pareto optimal solution (a (weak) efficient solution) in the following sense.

DEFINITION 2.3. A feasible point $\bar{x}$ is said to be a Pareto solution (efficient solution) for (VP) if and only if there exists no $x \in D$ such that $f(x) \leq f(\bar{x})$.

DEFINITION 2.4. A feasible point $\bar{x}$ is said to be a weak Pareto solution (weak efficient solution, weak minimum) for (VP) if and only if there exists no $x \in D$ such that $f(x)<f(\bar{x})$.

It is easy to verify that every Pareto solution is a weak Pareto solution.

It is well known (see, for example, $[9,13,17,19]$ ) that the Karush-Kuhn-Tucker conditions are necessary conditions for optimality in such optimisation problems.

THEOREM 2.5. Let $\bar{x}$ be a (weak) Pareto optimal solution in (VP) and let a suitable constraint qualification [4] be satisfied at $\bar{x}$. Then there exist $\bar{\lambda} \in R_{+}^{k}, \bar{\xi} \in R_{+}^{m}$, such that

$$
\begin{aligned}
\bar{\lambda} \nabla f(\bar{x})+\bar{\xi} \nabla g(\bar{x}) & =0, \\
\bar{\xi} g(\bar{x}) & =0, \\
\bar{\lambda} \geq 0, \quad \bar{\xi} & \geqq 0 .
\end{aligned}
$$




\section{An $\eta$-approximated vector optimisation problem and optimality conditions}

In [2], Antczak used the $\eta$-approximation approach for solving a differentiable scalar mathematical programming problem. Later, Antczak [3] extended the $\eta$ approximation approach to the vectorial case. In this approach one can obtain optimality conditions for Pareto optimality by constructing an equivalent vector optimisation problem for the considered multiobjective programming problem (VP) and then using an invexity concept in multiobjective programming. This equivalent vector optimisation problem is constructed using $\eta$-approximation of both the objective and constraint functions in the given feasible solution in the original multiobjective programming problem. Using this method Antczak established the equivalence between the original multiobjective programming problem and its associated $\eta$-approximated vector optimisation problem. Moreover, to prove this result he also assumed a suitable constraint qualification to be fulfilled for the considered multiobjective programming problem and some restrictions were imposed on the function $\eta$.

For the benefit of the reader, we recall the $\eta$-approximation approach introduced by Antczak [3] for solving a differentiable multiobjective programming problem. We now give a definition of the associated $\eta$-approximated vector optimisation problem $\left(\mathrm{VP}_{\eta}(\bar{x})\right)$ for the considered multiobjective programming problem (VP).

Let $\bar{x}$ be a given feasible solution in (VP). We construct the following vector optimisation problem $\left(\mathrm{VP}_{\eta}(\bar{x})\right)$ given by

$$
\begin{aligned}
& \left(f_{1}(\bar{x})+\nabla f_{1}(\bar{x}) \eta(x, \bar{x}), \ldots, f_{k}(\bar{x})+\nabla f_{k}(\bar{x}) \eta(x, \bar{x})\right) \rightarrow \min \\
& \text { subject to } \quad g_{j}(\bar{x})+\nabla g_{j}(\bar{x}) \eta(x, \bar{x}) \leqq 0, \quad j=1, \ldots, m,
\end{aligned}
$$

where $f, g, X$ are defined as in (VP) and $\eta$ is a vector-valued function defined by $\eta: X \times X \rightarrow R^{n}$. We denote by $\eta(\cdot, \bar{x})$ the function $x \rightarrow \eta(x, \bar{x})$. Throughout this paper we will assume that $\eta(\cdot, \bar{x})$ is a differentiable function at the point $x=\bar{x}$ with respect to the first component.

Antczak [3] established the equivalence between the vector optimisation problems (VP) and $\left(\mathrm{VP}_{\eta}(\bar{x})\right)$, that is, he proved that if $\bar{x}$ is a (weak) efficient point in (VP) then it is also (weak) efficient in $\left(\mathrm{VP}_{\eta}(\bar{x})\right)$, and also conversely, if $\bar{x}$ is a (weak) efficient point in $\left(\mathrm{VP}_{\eta}(\bar{x})\right)$ then it is also (weak) efficient in (VP). Further, optimality values are the same in both the considered vector optimisation problems (VP) and $\left(\operatorname{VP}_{\eta}(\bar{x})\right)$.

In [2], Antczak introduced the following restrictions imposed on the function $\eta$.

CONDITION A. We say that $\eta$ satisfies Condition A when $\eta(\cdot, \bar{x})$ is a differentiable function at the point $x=\bar{x}$ with respect to the first component and satisfies the following conditions: $\eta(\bar{x}, \bar{x})=0$ and $\eta_{x}(\bar{x}, \bar{x})=\alpha \cdot 1$, where $\eta_{x}(\bar{x}, \bar{x})$ denotes the derivative of $\eta(\cdot, \bar{x})$ at the point $x=\bar{x}$, and $\alpha$ is some positive real number. 
Note that the Karush-Kuhn-Tucker necessary optimality conditions for the $\eta$ approximated vector optimisation problem $\left(\mathrm{VP}_{\eta}(\bar{x})\right)$ have the following form.

THEOREM 3.1. Let $\bar{x}$ be a (weak) Pareto optimal solution in $\left(\mathrm{VP}_{\eta}(\bar{x})\right)$ and a suitable constraint qualification [4] be satisfied at $\bar{x}$. Then there exist $\bar{\lambda} \in R_{+}^{k}, \bar{\xi} \in R_{+}^{m}$, such that

$$
\begin{aligned}
(\bar{\lambda} \nabla f(\bar{x})+\bar{\xi} \nabla g(\bar{x})) \eta_{x}(\bar{x}, \bar{x}) & =0, \\
\bar{\xi}(g(\bar{x})+\nabla g(\bar{x}) \eta(\bar{x}, \bar{x})) & =0, \\
\bar{\lambda} \geq 0, \quad \bar{\xi} & \geqq 0 .
\end{aligned}
$$

REMARK 3.2. Antczak [3] proved that the Karush-Kuhn-Tucker necessary optimality conditions for the original multiobjective programming problem (VP) and its associated $\eta$-approximated vector optimisation problem $\left(\mathrm{VP}_{\eta}(\bar{x})\right)$ have the same form if the function $\eta$ is assumed to satisfy Condition $A$ at $\bar{x}$.

REMARK 3.3. In [3], Antczak introduced an $\eta$-approximation approach for solving a nonlinear differentiable multiobjective programming problem. The formulation of the introduced $\eta$-approximated vector optimisation problem requires the Lagrange multipliers of the original multiobjective programming problem (although, as follows from Remark 3.2, the Lagrange multipliers for an $\eta$-approximated vector optimisation problem are the same under Condition A). Thus apparently one cannot compute the $\eta$-approximated vector optimisation problem without first computing the original multiobjective programming problem. This follows from the construction of the introduced $\eta$-approximation approach, because we need a feasible point $\bar{x}$, which is suspected to be optimal. Then an $\eta$-approximated vector optimisation problem can be constructed at such a selected point. In general, we obtain a simpler vector optimisation problem to solve than the original nonlinear multiobjective programming problem. Hence, to solve the constructed (in most cases linear) vector optimisation problem, we can apply the known computational procedures. Moreover, there may exist more than one associated $\eta$-approximated vector optimisation problem (see Antczak [3]). These properties are also useful from the practical point of view. As follows from the above, we are in a position to solve strongly nonlinear nonconvex multiobjective programming problems by using computational procedures, for example, for solving linear vector optimisation problems.

\section{4. $\eta$-saddle point criteria}

In this section, we use the $\eta$-approximation method to obtain new saddle point criteria for differentiable multiobjective programming problems involving invex functions with respect to the same function $\eta$. 
We now introduce a definition of an $\eta$-Lagrange function for the constructed $\eta$-approximated vector optimisation problem $\left(\operatorname{VP}_{\eta}(\bar{x})\right)$.

DEFINITION 4.1. An $\eta$-approximated Lagrange function is said to be a Lagrange function for the vector optimisation problem $\left(\mathrm{VP}_{\eta}(\bar{x})\right)$

$$
\begin{aligned}
L_{\eta}(x, \lambda, \xi):= & \lambda f(\bar{x})+\xi g(\bar{x}) e+(\lambda \nabla f(\bar{x})+\xi \nabla g(\bar{x})) \eta(x, \bar{x}) \\
:= & \left(\lambda_{1} f_{1}(\bar{x})+\xi g(\bar{x})+(\lambda \nabla f(\bar{x})+\xi \nabla g(\bar{x})) \eta_{1}(x, \bar{x})\right. \\
& \left.+\cdots+\lambda_{k} f_{k}(\bar{x})+\xi g(\bar{x})+(\lambda \nabla f(\bar{x})+\xi \nabla g(\bar{x})) \eta_{k}(x, \bar{x})\right) \\
:= & L(\bar{x}, \lambda, \xi)+\nabla L(\bar{x}, \lambda, \xi) \eta(x, \bar{x}) .
\end{aligned}
$$

REMARK 4.2. Note that if the condition $\eta(\bar{x}, \bar{x})=0$ is fulfilled then, by Definition 4.1, $L_{\eta}(\bar{x}, \lambda, \xi)=L(\bar{x}, \lambda, \xi)$.

For the Lagrange function, some kinds of saddle points have been introduced (see, for example, $[7,20])$. Now, in a natural way, we introduce a definition for an $\eta$-saddle point for the $\eta$-Lagrange function in the $\eta$-approximated vector optimisation problem $\left(\mathrm{VP}_{\eta}(\bar{x})\right)$.

DEFINITION 4.3. A point $(\bar{x}, \bar{\lambda}, \bar{\xi}) \in D \times R_{+}^{k} \times R_{+}^{m}$ is said to be a (Pareto) $\eta$-saddle point for the $\eta$-approximated Lagrange function if

(i) $L_{\eta}(\bar{x}, \bar{\lambda}, \xi) \leqq L_{\eta}(\bar{x}, \bar{\lambda}, \bar{\xi})$ for all $\xi \in R_{+}^{m}$ and

(ii) $L_{\eta}(\bar{x}, \bar{\lambda}, \bar{\xi}) \leqq L_{\eta}(x, \bar{\lambda}, \bar{\xi})$ for all $x \in D$.

THEOREM 4.4. Let $f$ be invex (strictly invex) at $\bar{x}$ on D with respect to $\eta$ satisfying the following condition $\eta(\bar{x}, \bar{x})=0$. Moreover, we assume that some suitable constraint qualification is satisfied at $\bar{x}$ for (VP). If $(\bar{x}, \bar{\lambda}, \bar{\xi})$ is an $\eta$-saddle point for $L_{\eta}$ then $\bar{x}$ is a weak Pareto (Pareto) solution in (VP).

Proof. Let $(\bar{x}, \bar{\lambda}, \bar{\xi})$ be an $\eta$-saddle point for $L_{\eta}$. Then by Definition 4.3 (i) and Remark 4.2 we have

$$
\bar{\lambda} f(\bar{x})+\xi g(\bar{x}) \leqq \bar{\lambda} f(\bar{x})+\bar{\xi} g(\bar{x}), \quad \text { for all } \xi \in R_{+}^{m},
$$

and so

$$
\xi g(\bar{x}) \leqq \bar{\xi} g(\bar{x}), \quad \text { for all } \xi \in R_{+}^{m} .
$$

In (4.1), let $\xi=0$. Thus

$$
\bar{\xi} g(\bar{x}) \geqq 0 .
$$

We proceed by contradiction. Let us suppose that $\bar{x}$ is not a weak Pareto solution in (VP). Then there exists $\tilde{x} \in D$ such that

$$
f(\tilde{x})<f(\bar{x}) .
$$


Since $\bar{x} \in D(\bar{x})$ and $\bar{\xi} \in R_{+}^{m}$, we have

$$
\bar{\xi}(g(\bar{x})+\nabla g(\bar{x}) \eta(\bar{x}, \bar{x})) \leqq 0,
$$

and so

$$
\bar{\xi} g(\bar{x}) \leqq 0 .
$$

Hence, by (4.4) and (4.2),

$$
\bar{\xi} g(\bar{x})=0 .
$$

Thus, using the feasibility of $\tilde{x}$ in $\left(\mathrm{VP}_{\eta}(\bar{x})\right)$ together with (4.5), we get

$$
\bar{\xi} \nabla g(\bar{x}) \eta(\tilde{x}, \bar{x}) \leqq 0 .
$$

By assumption, $f$ is invex at $\bar{x}$ on $D$ with respect to $\eta$. Hence, by (4.3) and Definition 2.1, it follows that $\nabla f(\bar{x}) \eta(\bar{x}, \bar{x})<0$, and so, by $\bar{\lambda} \geq 0$,

$$
\bar{\lambda} \nabla f(\bar{x}) \eta(\tilde{x}, \bar{x}) \leq 0 .
$$

Thus, by (4.6) and (4.7) we obtain

$$
(\bar{\lambda} \nabla f(\bar{x})+\bar{\xi} \nabla g(\bar{x})) \eta(\tilde{x}, \bar{x}) \leq 0 .
$$

Now, using the definition of $L_{\eta}$, we get

$$
\begin{aligned}
L_{\eta}(\tilde{x}, \bar{\lambda}, \bar{\xi}) & =\bar{\lambda} f(\bar{x})+\bar{\xi} g(\bar{x}) e+(\bar{\lambda} \nabla f(\bar{x})+\bar{\xi} \nabla g(\bar{x})) \eta(\tilde{x}, \bar{x}) \\
& \leq \bar{\lambda} f(\bar{x})+\bar{\xi} g(\bar{x}) e+(\bar{\lambda} \nabla f(\bar{x})+\bar{\xi} \nabla g(\bar{x})) \eta(\bar{x}, \bar{x}) \\
& =L_{\eta}(\bar{x}, \bar{\lambda}, \bar{\xi})
\end{aligned}
$$

This contradicts Definition 4.3 (ii), provided $\bar{x}$ is a weak Pareto solution in (VP).

The proof of efficiency is similar.

We now prove a converse condition, that is, a sufficient condition for a point $(\bar{x}, \bar{\lambda}, \bar{\xi}) \in D \times R_{+}^{k} \times R_{+}^{m}$ to be an $\eta$-saddle point for the $\eta$-Lagrange function.

THEOREM 4.5. Let $\bar{x}$ be a (weak) Pareto optimal solution in (VP) and a suitable constraint qualification [4] be satisfied at $\bar{x}$. Further, we assume that $\eta$ satisfies the condition $\eta(\bar{x}, \bar{x})=0$. Then there exist $\bar{\lambda} \in R_{+}^{k}, \bar{\xi} \in R_{+}^{m}$, such that $(\bar{x}, \bar{\lambda}, \bar{\xi})$ is an $\eta$-saddle point for the $\eta$-Lagrange function in the $\eta$-approximated vector optimisation problem $\left(\mathrm{VP}_{n}(\bar{x})\right)$.

Proof. By assumption, $\bar{x}$ is a (weak) Pareto solution for (VP). Thus, by Theorem 2.5, it follows that the Karush-Kuhn-Tucker necessary optimality conditions (2.1) and (2.2) are satisfied. Then, by the Karush-Kuhn-Tucker condition (2.2), it 
follows that the inequality $\bar{\xi} g(\bar{x}) \geqq \xi g(\bar{x})$ holds for all $\xi \in R_{+}^{m}$. Then the assumption $\eta(\bar{x}, \bar{x})=0$ implies

$$
\begin{aligned}
& \bar{\lambda} f(\bar{x})+\xi g(\bar{x}) e+(\bar{\lambda} \nabla f(\bar{x})+\xi \nabla g(\bar{x})) \eta(\bar{x}, \bar{x}) \\
& \quad \leqq \bar{\lambda} f(\bar{x})+\bar{\xi} g(\bar{x}) e+(\bar{\lambda} \nabla f(\bar{x})+\bar{\xi} \nabla g(\bar{x})) \eta(\bar{x}, \bar{x}) .
\end{aligned}
$$

Hence from Definition 4.1 it follows that the inequality $L_{\eta}(\bar{x}, \bar{\lambda}, \xi) \leqq L_{\eta}(\bar{x}, \bar{\lambda}, \bar{\xi})$ is satisfied for all $\xi \in R_{+}^{m}$. This means that inequality (i) from Definition 4.3 is established.

We now prove the second inequality in Definition 4.3. Proof of this inequality follows directly from Definition 4.1 and by using the condition $\eta(\bar{x}, \bar{x})=0$ together with the Karush-Kuhn-Tucker optimality condition (2.3). Indeed, we have

$$
\begin{aligned}
L_{\eta}(x, \bar{\lambda}, \bar{\xi})-L_{\eta}(\bar{x}, \bar{\lambda}, \bar{\xi})= & \bar{\lambda} f(\bar{x})+\bar{\xi} g(\bar{x}) e+(\bar{\lambda} \nabla f(\bar{x})+\bar{\xi} \nabla g(\bar{x})) \eta(x, \bar{x}) \\
& -[\bar{\lambda} f(\bar{x})+\bar{\xi} g(\bar{x}) e+(\bar{\lambda} \nabla f(\bar{x})+\bar{\xi} \nabla g(\bar{x})) \eta(\bar{x}, \bar{x})] \\
= & (\bar{\lambda} \nabla f(\bar{x})+\bar{\xi} \nabla g(\bar{x})) \eta(x, \bar{x})=0 .
\end{aligned}
$$

Thus by Definition 4.3 we conclude that $(\bar{x}, \bar{\lambda}, \bar{\xi})$ is an $\eta$-saddle point for the $\eta$ Lagrange function in the $\eta$-approximated vector optimisation problem $\left(\mathrm{VP}_{\eta}(\bar{x})\right)$.

In view of Theorems 4.4 and 4.5 , we see that, if we assume that $f$ is (invex) strictly invex with respect to $\eta$ and $g$ is also invex at $\bar{x}$ on $D$ with respect to the same function $\eta$ satisfying $\eta(\bar{x}, \bar{x})=0$, and, moreover, some constraint qualification is satisfied at $\bar{x}$, then the $\eta$-approximation approach guarantees the equivalence between a (weak) Pareto solution $\bar{x}$ in (VP) and $\eta$-saddle points of the $\eta$-Lagrange function in its associated $\eta$-approximated vector optimisation problem $\left(\mathrm{VP}_{\eta}(\bar{x})\right)$ in the sense discussed above.

We now give an example of a multiobjective programming problem (VP) which by using the approach discussed in this paper is transformed to a less complicated vector optimisation problem $\left(\operatorname{VP}_{\eta}(\bar{x})\right)$. For the considered multiobjective programming problem we show the equivalence between its Pareto optimal solution $\bar{x}$ and the $\eta$ saddle points of its associated $\eta$-approximated vector optimisation problem $\left(\mathrm{VP}_{\eta}(\vec{x})\right)$.

EXAMPLE 1. We consider the following multiobjective programming problem:

$$
\begin{aligned}
& f(x)=\left(x^{4}+x^{2}-\frac{1}{5} \ln \left(x^{2}+1\right)+\frac{1}{2} e^{2 x}-\frac{1}{2}, x^{2} \arctan \left(x^{2}+1\right)+e^{2 x}\right) \rightarrow \min \\
& g(x)=-\arctan (2 x) \leqq 0 .
\end{aligned}
$$

Note that $D=\{x \in R: x \geqq 0\}$ and $\bar{x}=0$ is a Pareto optimal point in the considered multiobjective programming problem. It is not difficult to prove that $f$ and $g$ are 
strictly invex with respect to $\eta$ at $\bar{x}$ on $D$, for example, defined by

$$
\eta(x, \bar{x})=\left(e^{2 x}-e^{2 \bar{x}}\right) / 2 .
$$

Now, using the approach discussed in our paper we construct the associated vector optimisation problem $\left(\mathrm{VP}_{\eta}(\bar{x})\right)$ by transforming at $\bar{x}$ both the objective function $f$ and the constraint function $g$. Thus we obtain the following vector optimisation problem:

$$
\begin{aligned}
\left(\left(e^{2 x}-1\right) / 2,\left(e^{2 x}-1\right) / 2\right) & \rightarrow \min \\
1-e^{2 x} & \leqq 0 .
\end{aligned}
$$

It is not difficult to see that, similar to the original multiobjective programming problem, $\bar{x}=0$ is also a Pareto optimal solution in the above vector optimisation problem, that is, in the associated $\eta$-approximated vector optimisation problem $\left(\mathrm{VP}_{\eta}(\bar{x})\right)$. We now define the $\eta$-Lagrange function in $\left(\mathrm{VP}_{\eta}(\bar{x})\right)$. Then we have by Definition 4.1 that

$$
L_{\eta}(x, \lambda, \xi)=\left(\left(\lambda_{1} / 2-\xi\right)\left(e^{2 x}-1\right),\left(\lambda_{2} / 2-\xi\right)\left(e^{2 x}-1\right)\right) .
$$

It is not difficult to prove, by Definition 4.3 , that $(\bar{x}, \bar{\lambda}, \bar{\xi})=(0,2 k, k)$, where $k$ is any positive real number, is an $\eta$-saddle point in the constructed $\eta$-approximated vector optimisation problem $\left(\mathrm{VP}_{\eta}(\bar{x})\right)$. Since all hypothesis of Theorem 4.4 are fulfilled at $(\bar{x}, \bar{\lambda}, \bar{\xi})$ then, moreover, $\bar{x}=0$ is Pareto optimal in the original considered multiobjective programming problem. Thus we establish equivalence between the Pareto optimal solution $\bar{x}=0$ in the considered original multiobjective programming problem and the $\eta$-saddle points in its associated $\eta$-approximated vector optimisation problem. Moreover, as follows from this example, the introduced $\eta$-approximated approach allows us to solve the original multiobjective programming problem by the help of less complicated vector optimisation problem. It is sufficient to find $\eta$ saddle points of an $\eta$-Lagrange function for such problems. Note that the $\eta$-Lagrange function in the constructed $\eta$-approximated vector optimisation problem is also less complicated than the Lagrange function in the original multiobjective programming problem.

REMARK 4.6. Note that, in general, there exists more than one function $\eta$ with respect to which all functions involved in the original multiobjective programming problem are invex. This means that, in general, there exists more than one $\eta$-approximated vector optimisation problem associated with the original multiobjective programming problem. For example, it is not difficult to show that all functions involved in the multiobjective programming problem considered in Example 1 are also invex at $\bar{x}$ with respect to the same function $\eta$ defined by

$$
\eta(x, \bar{x})=\frac{1}{2} \arctan (2 x)-\frac{1}{2} \arctan (2 \bar{x}) .
$$


Obviously, this property of the introduced $\eta$-approximation approach is useful from a practical point of view.

REMARK 4.7. The assumption that a function $\eta$ satisfies the condition $\eta(\bar{x}, \bar{x})=0$ is essential to confirm equivalence between the vector optimisation problems (VP) and $\left(\mathrm{VP}_{\eta}(\bar{x})\right)$ in the sense discussed. In the example below we show that in the case when this condition does not hold then we have no equivalence between (VP) and $\left(\mathrm{VP}_{\eta}(\bar{x})\right)$.

We now give an example of a multiobjective programming problem in which all functions involved are invex with respect to the same function $\eta$. However, the function $\eta$ doesn't satisfy the condition $\eta(\bar{x}, \bar{x})=0$. In this case, we construct an associated $\eta$-approximated vector optimisation problem which is not equivalent to the original multiobjective programming problem in the sense discussed here. More exactly, we show that there is no equivalence between optima in the original multiobjective programming problem and the $\eta$-saddle points of the $\eta$-Lagrange function in its associated $\eta$-approximated vector optimisation problem.

EXAMPLE 2. We consider the following multiobjective programming problem:

$$
\begin{aligned}
& f(x)=\left(x_{1}^{2} x_{2}^{2},-x_{1}\right) \rightarrow \min \\
& g(x)=\left(x_{2}+1\right)^{2}-x_{1} \leqq 0 .
\end{aligned}
$$

Note that $D=\left\{\left(x_{1}, x_{2}\right) \in R^{2}: x_{1} \geqq 0 \wedge x_{2} \in R\right\}$ and $\bar{x}=(0,-1)$ is a weak Pareto optimal point in the considered multiobjective programming problem. It is not difficult to prove that $f$ and $g$ are strictly invex with respect to $\eta$ at $\bar{x}$ on $D$, for example, defined by

$$
\eta(x, \bar{x})=\left[\begin{array}{c}
x_{1}^{2}+x_{1}+1 \\
0
\end{array}\right] .
$$

Note that the function $\eta$ defined above doesn't satisfy the condition $\eta(\bar{x}, \bar{x})=0$. Now, using our approach we construct the associated vector optimisation problem $\left(\operatorname{VP}_{\eta}(\bar{x})\right)$ by transforming both the objective function $f$ and the constraint function $g$ at $\bar{x}$. Thus we obtain the following vector optimisation problem:

$$
\begin{gathered}
\left(0,-x_{1}^{2}-x_{1}-1\right) \rightarrow \min \\
x \in R^{2} .
\end{gathered}
$$

It is not difficult to see that $\bar{x}=(0,-1)$ is not a weak Pareto optimal solution in the above vector optimisation problem, that is, in the associated $\eta$-approximated vector 
optimisation problem $\left(\mathrm{VP}_{\eta}(\bar{x})\right)$. We now define the $\eta$-Lagrange function in $\left(\mathrm{VP}_{\eta}(\bar{x})\right)$. Then we have by Definition 4.1 that

$$
L_{\eta}(x, \lambda, \xi)=\left(0,-\lambda_{2}\left(x_{1}^{2}+x_{1}+1\right)\right) .
$$

It is not difficult to prove, by Definition 4.3 , that there is no $\bar{\lambda} \in R_{+}, \bar{\xi} \in R_{+},(\bar{\lambda}, \bar{\xi}) \neq$ $(0,0)$, such that $(\bar{x}, \bar{\lambda}, \bar{\xi})$ is an $\eta$-saddle point in the constructed $\eta$-approximated vector optimisation problem $\left(\mathrm{VP}_{\eta}(\bar{x})\right)$. Thus we show that there is no equivalence between the original multiobjective programming problem and its associated $\eta$-approximated vector optimisation problem $\left(\mathrm{VP}_{\eta}(\bar{x})\right)$. This follows from the fact that the function $\eta$ doesn't satisfy the condition $\eta(\bar{x}, \bar{x})=0$.

\section{Mond-Weir duality}

We now study the Mond-Weir-type duality [14] of the original multiobjective programming problem (VP) with the help of the Mond-Weir dual problem of the $\eta$-approximated vector optimisation problems, that is, the $\eta$-approximated Mond-Weir dual problems.

We consider the Mond-Weir-type dual of the primal multiobjective programming problem (VP):

$$
\begin{aligned}
V \text {-Maximise } & f(y) \\
\text { subject to } & \left\{\begin{array}{l}
\lambda \nabla f(y)+\xi \nabla g(y)=0 \\
\xi_{j} g_{j}(y) \geqq 0, \quad j=1, \ldots, m, \\
y \in X, \quad \lambda \geq 0, \quad \lambda e=1, \quad \xi \geqq 0 .
\end{array}\right.
\end{aligned}
$$

Note here that the symbol " $V$-Maximise" stands for vector (thus weak or Pareto) maximisation. We call (MWVD) the original Mond-Weir dual problem.

Let

$$
\begin{gathered}
W=\left\{(y, \lambda, \xi) \in X \times R_{+}^{k} \times R_{+}^{m}: \lambda \nabla f(y)+\xi \nabla g(y)=0,\right. \\
\left.\xi_{j} g_{j}(y) \geqq 0, j=1, \ldots, m, \lambda e=1\right\}
\end{gathered}
$$

denote the set of all feasible solutions in (MWVD). Further, let

$$
Y=\{y \in X:(y, \lambda, \xi) \in W\} .
$$

For the given feasible solution $(\tilde{y}, \tilde{\lambda}, \tilde{\xi}) \in W$ we construct $\left(\operatorname{VP}_{\eta}(\tilde{y})\right)$ and $\left(\operatorname{MWVD}_{\eta}(\tilde{y})\right)$ as follows:

$$
\begin{gathered}
f(\tilde{y})+\nabla f(\tilde{y}) \eta(x, \tilde{y}) \rightarrow \min \\
\text { subject to } g_{j}(\tilde{y})+\nabla g_{j}(\tilde{y}) \eta(x, \tilde{y}) \leqq 0, \quad j=1, \ldots, m, \\
x \in X .
\end{gathered}
$$




$$
\begin{aligned}
& f(\tilde{y})+\nabla f(\tilde{y}) \eta(y, \tilde{y}) \rightarrow \max \quad \text { subject to } \\
& \left\{\begin{array}{l}
\lambda \nabla f(\tilde{y})+\xi \nabla g(\tilde{y})=0, \\
\xi_{j}\left(g_{j}(\tilde{y})+\nabla g_{j}(\tilde{y}) \eta(y, \tilde{y})\right) \geqq 0, \quad j=1, \ldots, m, \\
y \in X, \quad \lambda \geq 0, \quad \lambda e=1, \quad \xi \geqq 0 .
\end{array}\right.
\end{aligned}
$$

We call (MWVD $(\tilde{y}))$ the $\eta$-approximated Mond-Weir dual problem at the given feasible point $(\tilde{y}, \tilde{\lambda}, \tilde{\xi})$.

We denote by $D(\tilde{y})$ and $W(\tilde{y})$ the sets of all feasible solutions in problems $\left(\operatorname{VP}_{\eta}(\tilde{y})\right)$ and $\left(\operatorname{MWVD}_{\eta}(\tilde{y})\right)$, respectively. Further, let $Y(\tilde{y})=\{y \in X:(y, \lambda, \xi) \in W(\tilde{y})\}$.

We now prove the weak duality theorem between the $\eta$-approximated vector optimisation problems $\left(\operatorname{VP}_{\eta}(\tilde{y})\right)$ and $\left(\operatorname{MWVD}_{\eta}(\tilde{y})\right)$.

PROPOSITION 5.1. Let $x$ and $(y, \lambda, \xi)$ be any feasible solutions in problems $\left(\mathrm{P}_{\eta}(\tilde{y})\right)$ and $\left(\mathrm{MWVD}_{\eta}(\tilde{y})\right)$, respectively. Then $\nabla f(\tilde{y}) \eta(x, \tilde{y}) \nless \nabla f(\tilde{y}) \eta(y, \tilde{y})$.

PROOF. Let $x$ and $(y, \lambda, \xi)$ be any feasible solutions in $\left(\mathrm{P}_{\eta}(\tilde{y})\right)$ and $\left(\mathrm{MWVD}_{\eta}(\tilde{y})\right)$, respectively. We proceed by contradiction. Suppose that

$$
\nabla f(\tilde{y}) \eta(x, \tilde{y})<\nabla f(\tilde{y}) \eta(y, \tilde{y}) .
$$

Hence, by $\lambda \geq 0, \lambda e=1$,

$$
\lambda \nabla f(\tilde{y}) \eta(x, \tilde{y}) \leq \lambda \nabla f(\tilde{y}) \eta(y, \tilde{y}) .
$$

Using the feasibility of $x$ in $\left(\mathrm{P}_{\eta}(\tilde{y})\right)$ together with $\xi \in R_{+}^{m}$ we obtain

$$
\xi g(\tilde{y})+\xi \nabla g(\tilde{y}) \eta(x, \tilde{y}) \leqq 0 .
$$

Since $(y, \lambda, \xi)$ is feasible in $\left(\operatorname{MWVD}_{\eta}(\tilde{y})\right)$, therefore, we have

$$
\xi g(\tilde{y})+\xi \nabla g(\tilde{y}) \eta(y, \tilde{y}) \geqq 0 .
$$

Thus, by (5.2) and (5.3), $\xi \nabla g(\tilde{y})(\eta(x, \tilde{y})-\eta(y, \tilde{y})) \leqq 0$. Using the first constraint of $\left(\operatorname{MWVD}_{\eta}(\tilde{y})\right)$, we obtain the inequality

$$
\lambda \nabla f(\tilde{y})(\eta(x, \tilde{y}) \geqq \lambda \nabla f(\tilde{y}) \eta(y, \tilde{y})),
$$

which contradicts (5.1). Thus we get the conclusion of this theorem.

Note that we established the weak duality theorem between $\left(\mathrm{VP}_{\eta}(\tilde{y})\right)$ and $\left(\operatorname{MWVD}_{\eta}(\tilde{y})\right)$ without any invexity assumption imposed on the functions involved in these problems.

Using the result above, we establish a weak duality theorem between the original problems (VP) and (MWVD). 
THEOREM 5.2. Let $\tilde{x}$ and $(\tilde{y}, \tilde{\lambda}, \tilde{\xi})$ be any feasible solutions in (VP) and (MWVD), respectively. Moreover, we assume that $f$ and $g$ are invex at $\tilde{y}$ on $D \cup Y$ with respect to $\eta$. Then $f(\tilde{x}) \nless f(\tilde{y})$.

ProOF. Let $\tilde{x}$ and $(\tilde{y}, \tilde{\lambda}, \tilde{\xi})$ be any feasible solutions in (VP) and (MWVD), respectively. We proceed by contradiction. Suppose that

$$
f(\tilde{x})<f(\tilde{y}) .
$$

For the given feasible solution $(\tilde{y}, \tilde{\lambda}, \tilde{\xi}) \in W$ we construct $\left(\operatorname{VP}_{\eta}(\tilde{y})\right)$ and $\left(\operatorname{MWVD}_{\eta}(\tilde{y})\right)$. Note that $\tilde{x}$ and $(\tilde{y}, \tilde{\lambda}, \tilde{\xi})$ are also feasible for $\left(\operatorname{VP}_{\eta}(\tilde{y})\right)$ and $\left(\operatorname{MWVD}_{\eta}(\tilde{y})\right)$. Then, using the weak duality theorem between problems $\left(\mathrm{VP}_{\eta}(\tilde{y})\right)$ and $\left(\mathrm{MWVD}_{\eta}(\tilde{y})\right)$ (Proposition 5.1), we get that the inequality $\nabla f(\tilde{y}) \eta(\tilde{x}, \tilde{y}) \nless \nabla f(\tilde{y}) \eta(y, \tilde{y})$ holds for all $y \in Y(\tilde{y})$. Thus, for $y=\tilde{y}$,

$$
\nabla f(\tilde{y}) \eta(\tilde{x}, \tilde{y}) \nless \nabla f(\tilde{y}) \eta(\tilde{y}, \tilde{y}) .
$$

By assumption, $\eta(\tilde{y}, \tilde{y})=0$. Then (5.6) gives

$$
\nabla f(\tilde{y}) \eta(\tilde{x}, \tilde{y}) \nless 0 .
$$

Since $f$ is invex at $\tilde{y}$ on $D \cup Y$ then $f(\tilde{x})-f(\tilde{y}) \geqq \nabla f(\tilde{y}) \eta(\tilde{x}, \tilde{y})$. Thus (5.5) gives the inequality $\nabla f(\tilde{y}) \eta(\tilde{x}, \tilde{y})<0$, which contradicts (5.7).

We now prove the strong duality theorem between the $\eta$-approximated vector optimisation problems $\left(\mathrm{VP}_{\eta}(\bar{x})\right)$ and $\left(\mathrm{MWVD}_{\eta}(\bar{x})\right)$, where $\bar{x}$ is a (weak) Pareto optimal point in $\left(\mathrm{VP}_{\eta}(\bar{x})\right)$, and it is therefore also a (weak) Pareto optimal point in (VP) (see, Antczak [3]).

PROPOSITION 5.3 (Strong duality theorem). Let $\bar{x}$ be a (weak) Pareto optimal point in problem $\left(\mathrm{VP}_{\eta}(\bar{x})\right)$ and suppose that a suitable constraint qualification [4] is satisfied at $\bar{x}$. Moreover, $f$ and $g$ are assumed to be invex at $\bar{x}$ on $D \cup Y$ with respect to $\eta$ and the function $\eta$ is assumed to satisfy $\eta(\bar{x}, \bar{x})=0$. Then there exist $\bar{\lambda} \geq 0, \bar{\lambda} e=1$, $\bar{\xi} \geqq 0$ such that $(\bar{x}, \bar{\lambda}, \bar{\xi})$ is a (weak) maximal solution in $\left(\mathrm{MWVD}_{\eta}(\bar{x})\right.$ ).

Proof. Since $\bar{x}$ is a (weak) Pareto optimal solution in problem $\left(\operatorname{VP}_{\eta}(\bar{x})\right)$ then there exist $\bar{\lambda} \geq 0, \bar{\lambda} e=1, \bar{\xi} \geqq 0$ such that the Karush-Kuhn-Tucker necessary optimality conditions (3.1)-(3.3) are satisfied. We show that $(\bar{x}, \bar{\lambda}, \bar{\xi})$ is feasible in $\left(\mathrm{MWVD}_{\eta}(\bar{x})\right)$. Using the Karush-Kuhn-Tucker optimality conditions (3.1)-(3.3) together with $\eta(\bar{x}, \bar{x})=0$, we get

$$
\begin{aligned}
\bar{\lambda} \nabla f(\bar{x})+\bar{\xi} \nabla g(\bar{x}) & =0, \\
\bar{\xi}(g(\bar{x})+\nabla g(\bar{x}) \eta(\bar{x}, \bar{x})) & =0 .
\end{aligned}
$$


Hence the inequalities above imply the feasibility of $(\bar{x}, \bar{\lambda}, \bar{\xi})$ in $\left(\operatorname{MWVD}_{\eta}(\bar{x})\right)$.

We now prove that $(\bar{x}, \bar{\lambda}, \bar{\xi})$ is optimal in $\left(\operatorname{MWVD}_{\eta}(\bar{x})\right)$. We proceed by contradiction. Suppose that $(\bar{x}, \bar{\lambda}, \bar{\xi})$ is not a weak maximal solution in $\left(\operatorname{MWVD}_{\eta}(\bar{x})\right)$. Then there exists $(\tilde{y}, \tilde{\lambda}, \tilde{\xi})$ feasible in $\left(\operatorname{MWVD}_{\eta}(\bar{x})\right)$ such that

$$
f(\bar{x})+\nabla f(\bar{x}) \eta(\tilde{y}, \bar{x})>f(\bar{x})+\nabla f(\bar{x}) \eta(\bar{x}, \bar{x}),
$$

and so

$$
\nabla f(\bar{x}) \eta(\tilde{y}, \bar{x})>\nabla f(\bar{x}) \eta(\bar{x}, \bar{x}) .
$$

But the inequality above contradicts weak duality between problems $\left(\mathrm{VP}_{\eta}(\bar{x})\right)$ and $\left(\mathrm{MWVD}_{\eta}(\bar{x})\right.$ ) (Theorem 5.2).

Now, using the established strong duality result between the $\eta$-approximated vector optimisation problems $\left(\mathrm{VP}_{\eta}(\bar{x})\right)$ and $\left(\mathrm{MWVD}_{\eta}(\bar{x})\right)$, we prove the strong duality theorem between the original vector optimisation problems (VP) and (MWVD).

THEOREM 5.4 (Strong duality theorem). Let $\bar{x}$ be a (weak) Pareto optimal point in (VP) and suppose that a suitable constraint qualification is satisfied at $\bar{x}$. Further, we assume that $f$ and $g$ are invex at $\bar{x}$ on $D \cup Y$ with respect to $\eta$ satisfying the condition $\eta(\bar{x}, \bar{x})=0$. Then there exist $\bar{\lambda} \geq 0, \bar{\lambda} e=1, \bar{\xi} \geqq 0$ such that $(\bar{x}, \bar{\lambda}, \bar{\xi})$ is a (weak) maximal solution in (MWVD).

PROOF. Since $\bar{x}$ is a (weak) Pareto optimal solution in (VP) then there exist $\bar{\lambda} \geq 0$, $\bar{\lambda} e=1, \bar{\xi} \geqq 0$ such that the Karush-Kuhn-Tucker optimality conditions (2.3)(2.5) are satisfied. We now show that $(\bar{x}, \bar{\lambda}, \bar{\xi})$ is feasible in $\left(\mathrm{VP}_{\eta}(\bar{x})\right)$ and that it is also feasible in $\left(\mathrm{MWVD}_{\eta}(\bar{x})\right)$. Since $\bar{x} \in D$ and $g$ is invex at $\bar{x}$ on $D \cup Y$ then $(\bar{x}, \bar{\lambda}, \bar{\xi})$ is feasible in $\left(\operatorname{VP}_{\eta}(\bar{x})\right)$. Using the Karush-Kuhn-Tucker optimality conditions together with the constraint of $\left(\mathrm{MWVD}_{\eta}(\bar{x})\right)$ we obtain that $(\bar{x}, \bar{\lambda}, \bar{\xi})$ is also feasible in $\left(\mathrm{MWVD}_{\eta}(\bar{x})\right)$. By [3, Theorem 16] we have that $\bar{x}$ is also (weak) Pareto optimal in $\left(\mathrm{VP}_{\eta}(\bar{x})\right)$. Now, using the strong duality theorem for $\eta$-approximated problems $\left(\operatorname{VP}_{\eta}(\bar{x})\right)$ and $\left(\mathrm{MWVD}_{\eta}(\bar{x})\right)$ (Proposition 5.3), it follows that $(\bar{x}, \bar{\lambda}, \bar{\xi})$ is optimal in $\left(\operatorname{MWVD}_{\eta}(\bar{x})\right.$ ). Since $(\bar{x}, \bar{\lambda}, \bar{\xi})$ is (weak) maximal in problem $\left(\operatorname{MWVD}_{\eta}(\bar{x})\right)$, and, moreover, by assumption $g$ is invex at $\bar{x}$ on $D \cup Y$ with respect to $\eta$, then $(\bar{x}, \bar{\lambda}, \bar{\xi})$ is also feasible in problem (MWVD). Thus the conclusion of this theorem follows by the weak duality theorem (Theorem 5.2).

Now, by the help of converse duality between the $\eta$-approximated optimisation problems $\left(\mathrm{VP}_{\eta}(\bar{y})\right)$ and $\left(\mathrm{MWVD}_{\eta}(\bar{y})\right)$, we prove converse duality between the original vector optimisation problems (VP) and (MWVD), respectively.

THEOREM 5.5 (Converse duality). Let $(\bar{y}, \bar{\lambda}, \bar{\xi})$ be a (weak) maximal solution in (MWVD) such that $g(\bar{y})=0$. Moreover, we assume that $f$ and $g$ are invex at $\bar{y}$ on 
$D \cup Y$ with respect to $\eta$ satisfying the condition $\eta(\bar{y}, \bar{y})=0$. Then $\bar{y}$ is (weak) Pareto optimal in (VP).

Proof. We now prove that $(\bar{y}, \bar{\lambda}, \bar{\xi})$ is also a (weak) maximal solution in $\left(\operatorname{MWVD}_{\eta}(\bar{y})\right)$. We proceed by contradiction. Suppose that $(\bar{y}, \bar{\lambda}, \bar{\xi})$ is not a weak maximal solution in $\left(\mathrm{MWVD}_{\eta}(\bar{y})\right)$. Then there exists $(y, \lambda, \xi)$ feasible in $\left(\mathrm{MWVD}_{\eta}(\bar{y})\right)$ such that

$$
f(\bar{y})+\nabla f(\bar{y}) \eta(\bar{y}, \bar{y})<f(\bar{y})+\nabla f(\bar{y}) \eta(y, \bar{y}) .
$$

Hence, by $\bar{\lambda} \geq 0, \bar{\lambda} e=1$ and $\eta(\bar{y}, \bar{y})=0$,

$$
\bar{\lambda} \nabla f(\bar{y}) \eta(y, \bar{y}) \geq 0 .
$$

Since $(y, \lambda, \xi)$ is feasible in $\left(\operatorname{MWVD}_{\eta}(\tilde{y})\right)$ then we have

$$
\xi \nabla g(\bar{y}) \eta(y, \bar{y}) \leq 0 .
$$

By assumption, $g(\bar{y})=0$. Thus (5.10) gives $\xi(g(\bar{y})+\nabla g(\bar{y}) \eta(y, \bar{y})) \leq 0$. But the inequality above contradicts the feasibility of $(y, \lambda, \xi)$ in $\left(\operatorname{MWVD}_{\eta}(\bar{y})\right)$.

We now show that $\bar{y}$ is also a weak Pareto optimal solution in (VP( $(\bar{y}))$, that is, in the following vector optimisation problem:

$$
\begin{aligned}
& f(\bar{y})+\nabla f(\bar{y}) \eta(x, \bar{y}) \rightarrow \min \quad \text { subject to } \\
& \left\{\begin{array}{l}
g_{j}(\bar{y})+\nabla g_{j}(\bar{y}) \eta(x, \bar{y}) \leqq 0, \quad j=1, \ldots, m, \\
x \in X .
\end{array}\right.
\end{aligned}
$$

We proceed by contradiction. Suppose that $\bar{y}$ is not weak Pareto optimal in $(\mathrm{P}(\bar{y}))$, that is, there exists $x$ feasible in (VP( $\bar{y})$ ) such that

$$
f(\bar{y})+\nabla f(\bar{y}) \eta(x, \bar{y})<f(\bar{y})+\nabla f(\bar{y}) \eta(\bar{y}, \bar{y}) .
$$

Using $\eta(\bar{y}, \bar{y})=0$ together with $\bar{\lambda} \geq 0, \bar{\lambda} e=1$, we get

$$
\bar{\lambda} \nabla f(\bar{y}) \eta(x, \bar{y}) \leq 0 .
$$

Since $(\bar{y}, \bar{\lambda}, \bar{\xi})$ is feasible in (MWVD) then $\bar{\xi} g(\bar{y}) \geqq 0$. Thus

$$
\bar{\xi} \nabla g(\bar{y}) \eta(x, \bar{y}) \leqq 0 .
$$

Thus by (5.11) and (5.12) we obtain the inequality

$$
(\bar{\lambda} \nabla f(\bar{y})+\bar{\xi} \nabla g(\bar{y})) \eta(x, \bar{y}) \leq 0,
$$


which contradicts the first constraint of (MWVD).

We now prove that $\bar{y}$ is also weak Pareto optimal in (VP). We proceed by contradiction. Suppose that $\bar{y}$ is not Pareto optimal in (VP), that is, there exists $x$ such that

$$
f(x)<f(\bar{y})
$$

By assumption, $f$ is invex at $\bar{y}$ on $D \cup Y$, therefore, by Definition 2.1,

$$
f(x)-f(\bar{y}) \geqq \nabla f(\bar{y}) \eta(x, \bar{y}),
$$

and so (5.13) gives

$$
\nabla f(\bar{y}) \eta(x, \bar{y})<0 .
$$

By assumption, $g$ is invex at $\bar{y}$ on $D \cup Y$. Thus any feasible solution $x$ in problem (VP) is also feasible in problem $(\mathrm{P}(\bar{y}))$. Since $\bar{y}$ is weak Pareto optimal in (VP( $\bar{y})$ ) then

$$
f(\bar{y})+\nabla f(\bar{y}) \eta(\bar{y}, \bar{y})<f(\bar{y})+\nabla f(\bar{y}) \eta t(x, \bar{y}),
$$

and so $\nabla f(\bar{y})(x, \bar{y})>0$, which contradicts (5.14).

\section{Acknowledgements}

The author is thankful to Prof. B. D. Craven for his helpful suggestions and valuable comments which have considerably improved the final version of this article.

\section{References}

[1] T. Antczak, "A new approach to multiobjective programming with a modified objective function", J. Global Optim. 27 (2003) 485-495.

[2] T. Antczak, "An $\eta$-approximation approach for nonlinear mathematical programming problems involving invex functions", Numer. Funct. Anal. Optim. 25 (2004) 423-438.

[3] T. Antczak, "An $\eta$-approximation method in vector optimization", Nonlinear Anal. 63 (2005) 225-236.

[4] M. S. Bazaraa, H. D. Sherali and C. M. Shetty, Nonlinear programming: theory and algorithms (John Wiley and Sons, New York, 1991).

[5] C. R. Bector, S. Chandra and M. V. Durgaprasad, "Duality in pseudolinear multiobjective programming", Asia-Pac. J. Oper. Res. 5 (1988) 150-159.

[6] B. D. Craven, "Invex functions and constrained local minima", Bull. Austral. Math. Soc. 24 (1981) 357-366. 
[7] B. D. Craven, "Quasimin and quasisaddlepoint for vector optimization", Numer. Funct. Anal. Optim. 11 (1990) 45-54.

[8] R. R. Egudo and M. A. Hanson, "Multi-objective duality with invexity", J. Math. Anal. Appl. 126 (1987) 469-477.

[9] G. Giorgi and A. Guerraggio, "The notion of invexity in vector optimization: smooth and nonsmooth case", in Generalized Convexity, Generalized Monotonicity (eds. J. P. Crouzeix, J. E. Martinez-Legaz and M. Volle), Proceedings of the Fifth Symposium on Generalized Convexity, Luminy, France, (Kluwer, Dordrecht, 1997).

[10] T. R. Gulati and N. Talaat, "Duality in nonconvex vector minimum problems", Bull. Austral. Math. Soc. 44 (1991) 501-509.

[11] M. A. Hanson, "On sufficiency of the Kuhn-Tucker conditions", J. Math. Anal. Appl. 80 (1981) 545-550.

[12] D. S. Kim, "Optimality conditions and duality theorems for multiobjective invex programs", $J$. Inform. Optim. Sci. 12 (1991) 235-242.

[13] D. T. Luc, Theory of vector optimization, Lecture Notes in Economics and Mathematical Systems 319 (Springer-Verlag, Berlin, New York, 1989).

[14] B. Mond and T. Weir, "Generalized concavity and duality", in Generalized concavity in optimization and economics (eds. S. Schaible and W. T. Ziemba), (Academic Press, New York, 1981) 263-279.

[15] V. Pareto, Course d'economie politique (Rouge, Lausanne, 1896).

[16] C. Singh, "Duality theory in multiobjective differentiable programming", J. Inform. Optim. Sci. 9 (1988) 231-240.

[17] C. Singh, "Optimality conditions in multiobjective differentiable programming", J. Optim. Theory Appl. 53 (1988) 115-123.

[18] T. Weir, "Proper efficiency and duality for vector valued optimization problems", J. Austral. Math. Soc. Ser. A 43 (1987) 21-34.

[19] T. Weir, B. Mond and B. D. Craven, “On duality for weakly minimized vector valued optimization problems", Optimization 17 (1986) 711-721.

[20] T. Weir and B. Mond, "Pre-invex functions in multiple objective optimization", J. Math. Anal. Appl. 136 (1988) 29-38. 